\title{
Optical control of vibrational coherence triggered by an ultrafast phase transition
}

\author{
M. J. Neugebauer, ${ }^{1, *}$ T. Huber, ${ }^{1}$ M. Savoini, ${ }^{1}$ E. Abreu, ${ }^{1}$ V. Esposito, ${ }^{2}$ M. Kubli, ${ }^{1}$ L. Rettig,,${ }^{2, \dagger}$ E. Bothschafter, ${ }^{2}$ S. Grübel, ${ }^{2}$ \\ T. Kubacka, ${ }^{1}$ J. Rittmann, ${ }^{2}$ G. Ingold, ${ }^{2}$ P. Beaud, ${ }^{2}$ D. Dominko, ${ }^{3,4}$ J. Demsar, ${ }^{3}$ and S. L. Johnson ${ }^{1}$ \\ ${ }^{1}$ Institute for Quantum Electronics, Physics Department, ETH Zurich, CH-8093 Zurich, Switzerland \\ ${ }^{2}$ Swiss Light Source, Paul Scherrer Institut, CH-5232 Villigen PSI, Switzerland \\ ${ }^{3}$ Institute for Physics, Johannes Gutenberg Universität Mainz, D-55128 Mainz, Germany \\ ${ }^{4}$ Institute of Physics, HR-10000 Zagreb, Croatia
}

(Received 1 February 2019; revised manuscript received 5 April 2019; published 7 June 2019)

\begin{abstract}
Femtosecond time-resolved x-ray diffraction is employed to study the dynamics of the periodic lattice distortion (PLD) associated with the charge-density wave in $\mathrm{K}_{0.3} \mathrm{MoO}_{3}$. Using a multipulse scheme we show the ability to extend the lifetime of coherent oscillations of the PLD about the undistorted structure through reexcitation of the electronic states. This suggests that it is possible to enter a regime where the symmetry of the potential energy landscape corresponds to the high-symmetry phase but the scattering pathways that lead to the damping of coherent dynamics are still controllable by altering the electronic state population. The demonstrated control over the coherence time offers different routes for the manipulation of coherent lattice states.
\end{abstract}

DOI: 10.1103/PhysRevB.99.220302

The use of ultrashort laser pulses to generate and manipulate coherent states of lattice vibrations has been demonstrated in a wide variety of crystalline materials $[1,2]$. Typically, the largest responses are obtained when the pulse photon energy is tuned to a region of pronounced absorption in the material, triggering electronic transitions that strongly couple to small wave-vector vibrational modes. This is often referred to as "displacive excitation of coherent phonons" (DECP), in the limit where the light absorption happens on timescales shorter than the period of resulting vibrations [3-5]. The DECP mechanism is often understood in terms of a time-dependent interatomic potential energy surface for the crystal ions. The fast absorption induces a sudden shift in the quasiequilibrium structure of the crystal which excites a coherent oscillation of a normal mode about a displaced coordinate. Several experiments have demonstrated coherent control of these oscillations in different materials using a multipulse scheme to further shift the quasiequilbrium structure at controlled time delays [6-10].

Strong optical excitation can lead to changes in the overall symmetry of the interatomic potential, a phenomenon that is often identified as an "ultrafast phase transition" [11-16]. In some cases the symmetry change is short lived and collapses back into the low symmetry within a few picoseconds [17]. In this situation multiple pulse excitation enables the study of the dynamically evolving potential surface by inducing DECP in the partially relaxed structure [18]. In other cases, under strong enough excitation conditions and/or long-lived electronic and structural excitations, the change in symmetry persists up to microseconds. Typically, the system then relaxes back to the low-symmetry state only after thermalization and

\footnotetext{
*mj.neugebauer@phys.ethz.ch

$\dagger$ Present address: Abteilung Physikalische Chemie, Fritz-HaberInstitut der Max-Planck-Gesellschaft, D-14195 Berlin, Germany.
}

heat transport have led to cooling the material back to its initial temperature. Several experiments have studied this regime and observed dynamics in the high-symmetry structural configuration [14-16]. Beyond that the possibility of controlling coherent oscillations within the high-symmetry state remains largely unexplored. Here, we focus on this issue, exploring possible avenues of control over the dynamics that follow the light-driven collapse of the charge-density-wave (CDW) order in $\mathrm{K}_{0.3} \mathrm{MoO}_{3}$, a model system for a one-dimensional Peierls transition [19].

In equilibrium, $\mathrm{K}_{0.3} \mathrm{MoO}_{3}$ undergoes a metal-to-insulator transition at $T_{\mathrm{c}}=183 \mathrm{~K}$, accompanied by the formation of a CDW [20-22]. Strong excitation with a femtosecond optical pulse can melt the CDW, inducing a phase transition to the high-symmetry state. Experiments using optical reflectivity as a probe show either a disappearance of amplitude mode oscillations [23] or a dramatic softening and increase in damping [24] above a critical absorbed fluence of $F_{\mathrm{c}}^{\mathrm{opt}} \approx 0.3 \mathrm{~mJ} / \mathrm{cm}^{2}$ for pump pulses at a wavelength $\lambda=800 \mathrm{~nm}$. Experiments using $\mathrm{x}$ rays to probe directly the collapse of the periodic lattice distortion (PLD) estimate a critical fluence of $F_{\mathrm{c}}^{\mathrm{x} \text {-ray }} \approx$ $1.0 \mathrm{~mJ} / \mathrm{cm}^{2}$ [14], which is roughly comparable to $F_{\mathrm{c}}^{\mathrm{opt}}$, especially considering differences in the probing methods. For excitation fluences $F \geqslant 1.5 F_{\mathrm{c}}^{\mathrm{x} \text {-ray }}$ the PLD does not simply vanish but transiently revives after around $0.3 \mathrm{ps}$, which is ascribed to coherent dynamics along the Peierls coordinate [14]. These dynamics correspond to a pair of acoustic modes with the wave vector of the Peierls distortion but in a quasiequilibrium structure with symmetry equivalent to the metallic phase. The coherent dynamics exhibit an unusual damping behavior, resulting in an abrupt stop of coherent motion after only half a vibrational period. This appears to be inconsistent with the normal assumption of viscous damping [14].

These observations open the question of whether some degree of control of these coherent dynamics in the highsymmetry phase is possible, despite the fact that the high wave 


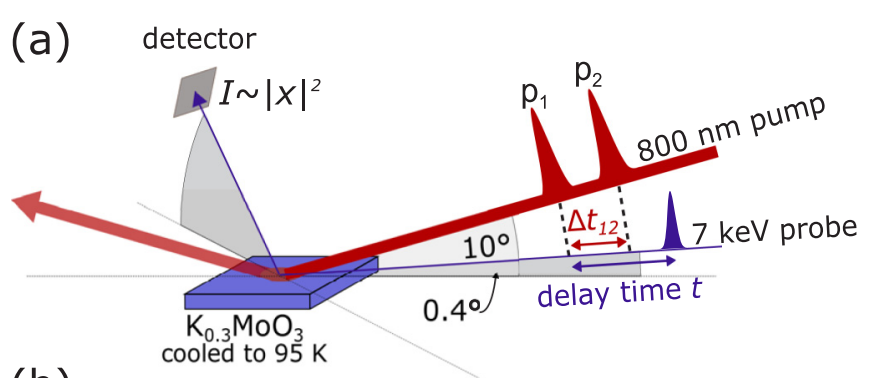

(b)

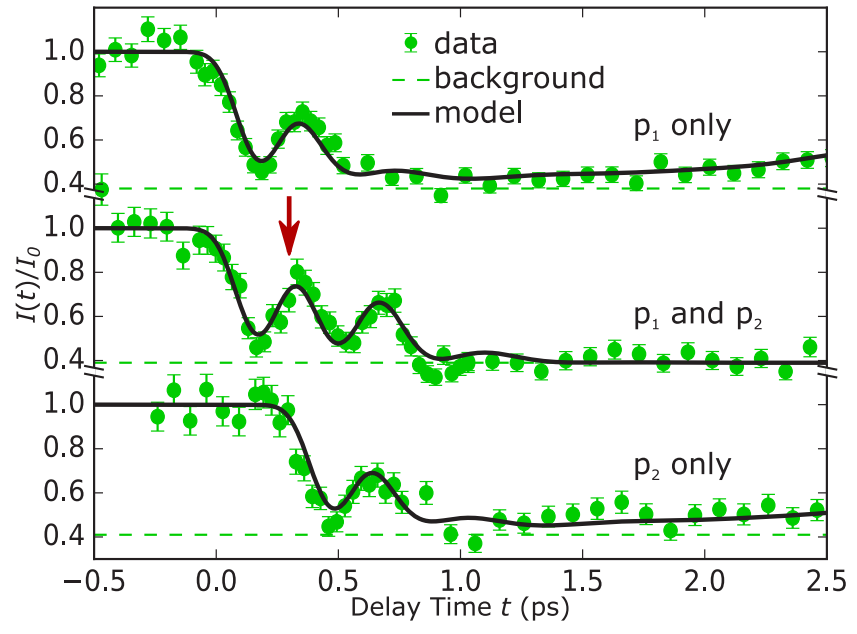

FIG. 1. Scheme of the experimental setup in (a) and comparison between individual and sequential application of the pump pulses in (b). The two different delay times are indicated in (a). One is the delay time $t$ between the first pump pulse $p_{1}$ and the x-ray probe, while $\Delta t_{12}$ is the time delay between the two pump pulses. In (b) the trace with the double-pulse excitation (middle) is compared to the traces obtained by excitations with individual $p_{1}$ (top) and $p_{2}$ (bottom) pulses at $F_{1}=F_{2}=F_{0}$. Dashed lines indicate the background level, and black lines correspond to the model (cf. text).

vector of the underlying acoustic modes normally precludes further displacive optical excitation. We explore this question using a two-pulse excitation scheme, where the first pump melts the electronic order and launches the coherent motion, and the second reexcites the system during the motion. We study with time-resolved $\mathrm{x}$-ray diffraction how the reexcitation of the second pulse affects the coherent dynamics.

For our experiments we use a bulk sample of $\mathrm{K}_{0.3} \mathrm{MoO}_{3}$ cleaved along its (201) plane and cooled with a nitrogen blower to $95 \mathrm{~K}$, substantially below $T_{\mathrm{c}}$. The PLD associated with the CDW can be probed using hard $\mathrm{x}$-ray diffraction by monitoring the intensity of the $\left(1\left(4-q_{b}\right) \overline{0.5}\right)$ superlattice Bragg reflection, where $q_{\mathrm{b}}$ is the modulation wave vector along the chain direction ( $b$ axis). At $100 \mathrm{~K}$ the modulation wave vector is $q_{\mathrm{b}}=0.748$ (1) [25]. In the kinematic approximation the diffraction intensity is proportional to the square of the magnitude of the PLD.

A sketch of the experimental setup is presented in Fig. 1(a). The structural dynamics associated with the CDW state are investigated using 7-keV $\mathrm{x}$-ray pulses with a full width at half maximum (FWHM) duration of around 120 fs and the sample is excited with 100-fs (FWHM) p-polarized 800-nm laser pulses. With the grazing geometry used this results in penetration depths of $\delta_{\mathrm{L}}=80 \mathrm{~nm}$ for the 800 -nm pump and $\delta_{\mathrm{X}}=100 \mathrm{~nm}$ for the $\mathrm{x}$ rays [14]. A Mach-Zehnder scheme creates a second pump pulse $p_{2}$, which can be delayed by $\Delta t_{12}$ relative to the first pump pulse $p_{1}$. In order to match the penetration depths of the optical and $\mathrm{x}$-ray beams a grazing incidence geometry is chosen. We set $F_{1}$ to $F_{0}=1.7 \mathrm{~mJ} / \mathrm{cm}^{2}$ to be above the critical fluence of the previous study [14], while $F_{2}$ varies between $F_{0} / 4$ and $F_{0}$. We estimate the experimental time resolution to be 150 fs [26].

Figure 1(b) shows the time evolution of the superlattice diffraction intensity for excitation with each pulse individually as well as both sequentially. If only $p_{1}$ or $p_{2}$ are applied at a fluence of $F_{0}=1.7 \mathrm{~mJ} / \mathrm{cm}^{2}$, a single transient revival appears $0.30 \mathrm{ps}$ after the arrival of the excitation pulse, in agreement with the results of Ref. [14] (cf. Fig. 1 of Ref. [26]). The middle plot shows the time evolution when both $p_{1}$ and $p_{2}$ are present and $\Delta t_{12}=0.30 \mathrm{ps}$ (the arrival of $p_{2}$ is indicated with a red arrow in all plots). Here, a second revival of the CDW distortion is visible at $t \approx 0.60 \mathrm{ps}$, whose shape and magnitude resemble the first one. A background level intensity $I_{\text {bg }}$ remains in the superlattice diffraction peak even for high excitation fluence. We ascribe this to the fraction of unexcited volume of the sample that is probed by the $\mathrm{x}$ rays [14]. In all plots the background level $I_{\text {bg }}$ fit to the model curves is shown as a dashed line.

We now focus on the temporal evolution of the PLD as a function of the reexcitation delay $\Delta t_{12}$ between 0.18 and 1.00 ps with $F_{2}=F_{1}$, as shown in Fig. 2(a). Clearly, the magnitude of the second revival depends on $\Delta t_{12}$, with a maximum near $\Delta t_{12}=0.30$ ps. A further increase of $\Delta t_{12}$, e.g., to $\Delta t_{12}=0.50$ or $1.00 \mathrm{ps}$, leads to no clear additional response of the system.

We also varied $F_{2}$ while keeping $\Delta t_{12}$ at $0.30 \mathrm{ps}$. The resulting delay time scans for $F_{2}=F_{1}, F_{1} / 2$, and $F_{1} / 4$ are displayed in Fig. 2(b). We define the amplitude of the first revival $A_{1}$ as the difference between its maximum and the minimum of the first half cycle, and the second revival amplitude $A_{2}$ accordingly. The ratio of $A_{2} / A_{1}$ scales linearly with $F_{2}$, as shown in the inset. Additionally, we show $A_{2} / A_{1}$ for $\Delta t_{12}=0.30 \mathrm{ps}$ and $F_{2}=F_{1}$ from the other two data sets [cf. Figs. 1 and 2(a), colors correspond] to emphasize the similar amplitude of the two revivals for this configuration. The timing of the revivals are, within our experimental uncertainties, independent of $\Delta t_{12}$ and $F_{2}$.

To describe the dynamics we extend the phenomenological model of Ref. [14]. The concept is similar to that of the Landau theory for second-order phase transitions [27], where we define a parametrization of an effective ionic potential energy surface rather than a free energy. The basic idea is that the shape of the effective potential depends strongly on the electronic states that are populated at a given time after the optical excitation. For simplicity we will consider a potential

$$
V(x)=\frac{1}{2} a x^{2}+\frac{1}{4} b x^{4}
$$

where $a$ and $b$ are parameters, and $x$ is a structural coordinate giving the instantaneous magnitude of the PLD associated with the CDW. As in Ref. [14], we consider the parameter $a$ to be a function of the electronic state of the material and the parameter $b$ to be constant. For convenience we will work in dimensionless units for $V$ and $x$, where $b=1$ and $a=-1$ for 

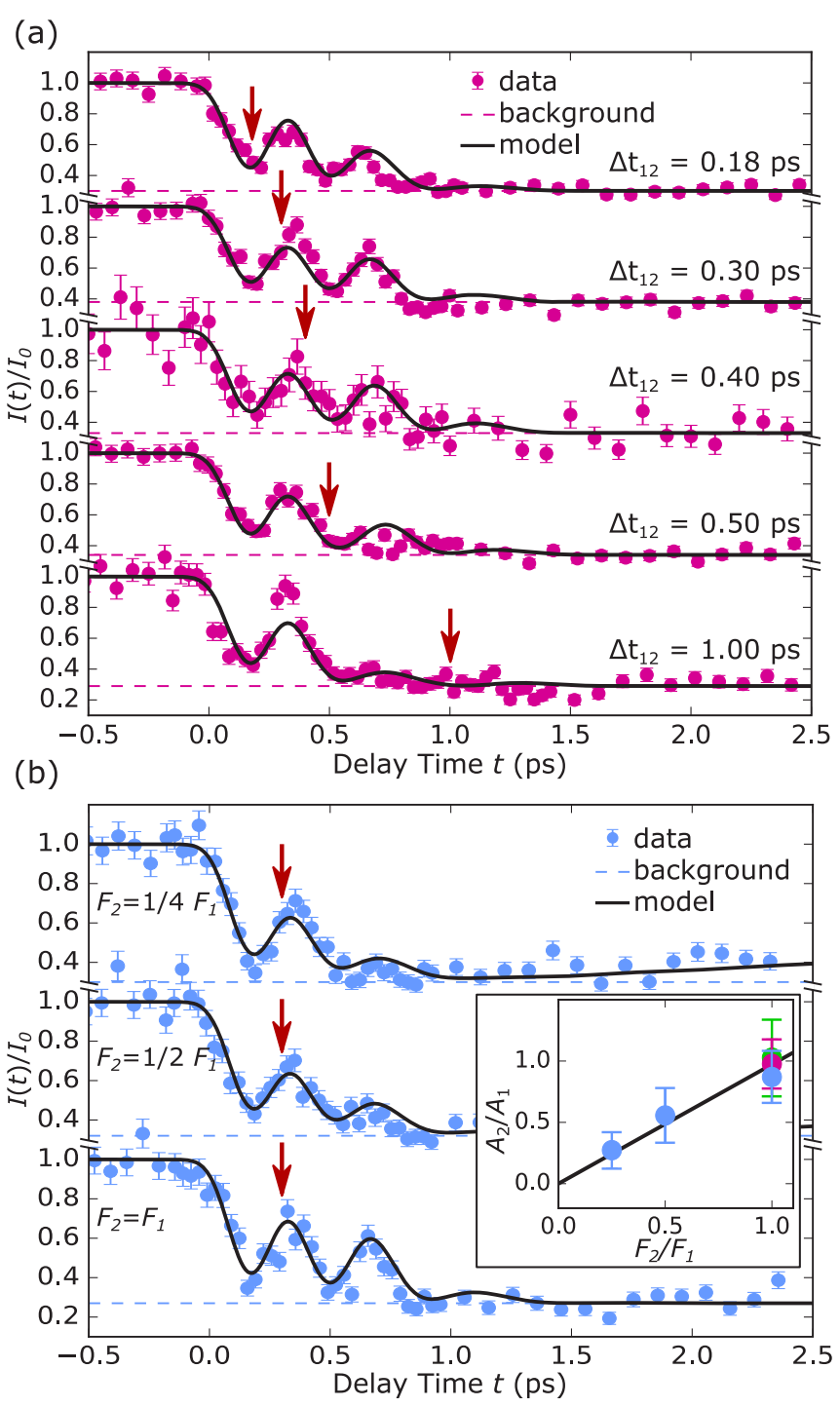

FIG. 2. The data taken in the double-pulse excitation configuration. (a) The dependence on $\Delta t_{12}$ for fixed $F_{1}=F_{2}=F_{0}$. (b) The dependence on $F_{2}$ for fixed $\Delta t_{12}=0.30 \mathrm{ps}$. Dashed lines indicate the background level, and black lines correspond to the model (cf. text). The inset of (b) shows the dependence of the ratio between the amplitude of the second $\left(A_{2}\right)$ and the first $\left(A_{1}\right)$ revival on the relative fluence of the second excitation for $\Delta t_{12}=0.30 \mathrm{ps}$ including a linear fit. The colors of the data points indicate the corresponding time trace (see also Fig. 1).

the ground state of the material. For these choices, the minima of $V(x)$ in the ground state occur at $x_{\min }= \pm 1$. Without loss of generality we assume that the equilibrium state is at $x_{0}=1$. For a more general value of $a$ we have either $x_{\min }= \pm \sqrt{a}$ for $a<0$ or $x_{\min }=0$ for $a \geqslant 0$. We can identify $x_{\min }$ as an effective order parameter of the CDW phase.

The electronic excitation of the material from the laser interaction will cause $a$ to become time dependent. In Ref. [14], $a$ was assumed to depend linearly on a dimensionless electronic energy density parameter $\eta$. While this may be appropriate for low or moderate excitation levels, at high excitation levels we encounter a problem since allowing an arbitrarily large value of $a$ gives unrealistically high frequencies for vibrations along the PLD coordinate $x$ for strong excitation levels. We will therefore make a rough approximation for $a(\eta)$ that prevents this effect by defining

$$
a(\eta)= \begin{cases}\eta-1, & \text { if } \eta<1+a_{\max }, \\ a_{\max }, & \text { if } \eta \geqslant 1+a_{\max },\end{cases}
$$

where $a_{\max }>0$ is a constant.

The excitation parameter $\eta$ depends on time, depth $z$ from the sample surface, and the strength of the pump pulse(s). For a single excitation pulse at $t=0$, we approximate $\eta$ as

$$
\eta_{\mathrm{S}}(z, t)=\eta_{0} e^{-z / \delta_{L}} e^{-t / \tau_{\text {disp }}} \Theta(t),
$$

where $\eta_{0}$ is a dimensionless parameter depending on the pump fluence $F_{1}, \delta_{\mathrm{L}}$ is the $1 / e$ penetration depth of the laser intensity, $\tau_{\text {disp }}$ is a relaxation time, and $\Theta$ is the Heaviside step function. If we now add a second pulse with fluence $F_{2}$ separated by a time $\Delta t_{12}$, we have instead

$$
\begin{aligned}
\eta_{\mathrm{D}}(z, t)= & \eta_{0} e^{-z / \delta_{\mathrm{L}}}\left[\Theta(t) e^{-t / \tau_{\text {disp }}}\right. \\
& \left.+\Theta\left(t-\Delta t_{12}\right) \frac{F_{2}}{F_{1}} e^{-\left(t-\Delta t_{12}\right) / \tau_{\text {disp }}}\right] .
\end{aligned}
$$

The duration of the excitation pulses is taken into account by a convolution with Gaussian of 0.10 ps FWHM.

The equation of motion for $x$ is

$$
\ddot{x}=-\omega^{2}\left[a(t) x+x^{3}\right]-2 \gamma(t) \dot{x},
$$

where $\omega=2 \pi v, v=1.53 \mathrm{THz}$ is the amplitude mode frequency in the ground state [14], and $\gamma(t)$ is a phenomenological damping coefficient. As discussed in Ref. [14], in order to make it possible to fit Eq. (5) to the dynamics we observe experimentally, $\gamma$ should be suppressed for a short time after the pulse. Microscopically, this would correspond to fewer scattering channels from the amplitude mode available under conditions of very high electronic excitation. Using arguments analogous to our form for $a(\eta)$, we consider this transient suppression of damping to be of the form

$$
\gamma(z, t)= \begin{cases}\gamma^{*}(z, t), & \text { if } \gamma^{*}(z, t)>\gamma_{\min }, \\ \gamma_{\min }, & \text { otherwise }\end{cases}
$$

with

$$
\begin{aligned}
\gamma^{*}(z, t)= & \gamma_{\text {unex }} \Theta(-t)+\gamma_{\text {sat }}\left[1-g(a+1) e^{-z / \delta_{\mathrm{L}}}\left(\Theta(t) e^{-t / \tau_{\gamma}}\right.\right. \\
& \left.\left.+\frac{F_{2}}{F_{1}} \Theta\left(t-\Delta t_{12}\right) e^{-\left(t-\Delta t_{12}\right) / \tau_{\gamma}}\right)\right]
\end{aligned}
$$

where $g$ is a dimensionless constant and $\tau_{\gamma}$ is a relaxation timescale. The constants $\gamma_{\text {unex }}$ and $\gamma_{\text {min }}$ are introduced as the damping value before excitation and the minimum value for the transient damping parameter, respectively. Physically, $\gamma_{\text {min }}$ represents alternative scattering channels that are not suppressed by the electronic excitation. We set $\gamma_{\text {unex }}$ to $0.4 \mathrm{ps}^{-1}$ and $\gamma_{\min }$ to $0.2 \mathrm{ps}^{-1}$ - see Ref. [26]. We can now solve Eq. (5) with initial conditions $x=x_{0}$ and $\dot{x}=0$ to find $x$ as a function of both time $t$ and depth $z$.

The intensity of x-ray diffraction from the superlattice peak is proportional to a weighed average of $x(z, t)$ over the $1 / e$ 


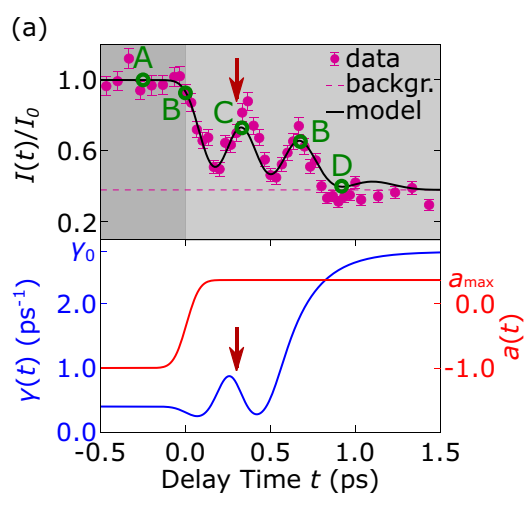

(b)

FIG. 3. Visualization of the model described in the text. The upper curve of (a) is a typical pump-probe trace with $\Delta t_{12}=300 \mathrm{fs}$ and $F_{2}=F_{1}$ [dashed lines indicate the background level, and black lines correspond to the model (cf. text)]. Below the corresponding progression of the damping $\gamma$ (blue, left axis) and $a(t)$ (red, right axis) near the surface are shown. (b) Sketch of the evolution of the PLD in its potential at delay times denoted on the pump-probe trace. The background colors indicate the potential configuration the system is in at different delay times.

attenuation length $\delta_{\mathrm{X}}=100 \mathrm{~nm}$ of the $\mathrm{x}$-ray intensity

$$
\frac{I(t)}{I_{0}}=\frac{1}{\delta_{\mathrm{X}}} \int_{0}^{\infty} x^{2}(z, t) e^{-2 z / \delta_{\mathrm{X}}} d z
$$

which we then convolve with a Gaussian of FWHM 150 fs to approximate the experimental time resolution.

The top part of Fig. 3(a) shows a fit from this model compared to data with $\Delta t_{12}=0.30 \mathrm{ps}$ and $F_{2}=F_{1}$, while the bottom part displays the time evolution of $\gamma(t)$, and $a(t)$ at $z=$ 0 . A sketch of the time-dependent potential energy surface is depicted in Fig. 3(b). The letters A-D guide through the measured pump-probe dynamics relating the corresponding points in the potential landscape, while the background colors mark the current effective potential configuration. In the beginning the system is in its double-well equilibrium state at A. At $t=0$ the first pump pulse $p_{1}$ arrives, promotes $a(t)$ to $a_{\max }$ (B), and quenches $\gamma(t)$ from $\gamma_{\text {unex }}$ to $\gamma_{\min }$. The system then goes through the minimum and overshoots to the opposite side of the high-energy potential. At $t=0.30 \mathrm{ps}, p_{2}$ excites the system again (C), only suppressing the damping $\gamma(t)$ without changes in $V$. Afterwards, the system swings back to $\mathrm{B}$, and finally comes to a stop in the single-well minimum at $\mathrm{D}$, since the damping has reached its saturation value $\gamma_{\text {sat }}$.

We fit all presented data sets with four global parameters, namely, $a_{\max }, \gamma_{\mathrm{sat}}, g$, and $\tau_{\gamma}$, while $\eta_{0}$ and $\tau_{\text {disp }}$ are determined only for the data sets showing a partial recovery within the monitored time frame [26]. $I_{\mathrm{bg}}$ is fit for each curve individually [26]. The parameter $\gamma_{\mathrm{sat}}=2.81 \pm 0.38 \mathrm{ps}^{-1}$ is similar to the damping constant close to the thermal transition [26], whereas $\tau_{\gamma}=0.18 \pm 0.11 \mathrm{ps}$ is comparable to the fast relaxation time of Ref. [23]. The resulting model curves are shown in all figures as black solid lines. With a small set of fit parameters our model reproduces the overall features of all data sets, including the single pump time traces at various fluences from Ref. [14] [cf. Fig. S1(a) in Ref. [26]]. The observation of the current double-pump data is that a second revival is present only when a second excitation arrives while the coherent motion after the first pump pulse still persists. This is reproduced by our simple model, which describes the qualitative dynamics of the system quite consistently. This is true for both the absence of a second PLD revival for $\Delta t_{12}=1.00 \mathrm{ps}$ and the scaling of $A_{2}$ for different values of $F_{2}$ as presented in Fig. 2(b).

The appearance of a second revival in the case of an additional pump between $\Delta t_{12}=0.18$ and $0.40 \mathrm{ps}$ unambiguously identifies this phenomenon as coherent PLD oscillations in the photoinduced high-symmetry phase. As mentioned above, the timing of the second revival is independent of changes in the timing and strength of the second pump pulse. This suggests that the frequency of the vibrational mode is not strongly changed by the second pulse.

When comparing the presented PLD dynamics in the highsymmetry phase to the doubly pumped coherent structural dynamics of materials far from a phase transition, such as the coherently driven $A_{1 g}$ mode of bismuth at low excitation fluences [6,9], a different behavior is observed. Here, the symmetry of the potential energy surface is unchanged, allowing the second pulse to further shift the values of $x_{\min }$ at well-defined times $t$ after the initial DECP. This enables a selective enhancement or cancellation of the coherent phonon, since the effect of the second excitation depends on the phase of $x(t)$. We observe something fundamentally different in the high excitation limit: The first pulse already changes the symmetry of the potential energy surface to that of the undistorted phase, and the second pulse cannot further shift $x_{\min }$ displacively. It does, however, influence the dynamics by extending the time over which underdamped dynamics occur. The mechanism behind the damping evolution is unclear, and could be either the result of a suppression of electronphonon coupling channels or the modulation of anharmonic coupling to other vibrational modes. Methods such as timeand angle-resolved photoelectron emission spectroscopy or nonequilibrium diffuse scattering could help to shed light on the details of the damping mechanism.

Time-resolved x-ray diffraction measurements were performed at the X05LA, and preparative static grazing incidence diffraction measurements were conducted at the X04SA beam lines of the Swiss Light Source, Paul Scherrer Institut, Villigen. We thank P. Willmott and D. Grolimund for experimental help and L. Huber and G. Lantz for discussions. We acknowledge funding through the NCCR Molecular Ultrafast Science and Technology (NCCR MUST), a research instrument of the Swiss National Science Foundation (SNSF). E.A. acknowledges support from the ETH Zurich Postdoctoral Fellowship and the Marie Curie Actions for People COFUND Programs, E.B. from the European Community's Seventh Framework Programme (FP7/2007-2013) under Grant Agreement No. 290605 (PSI-FELLOW/COFUND), and D.D. from the FemtoBias project, the Grant Agreement 55 of the NEWFELPRO fellowship project (Grant Agreement No. 291823) cofinanced by MSCA-FP7-PEOPLE-2011-COFUND. 
[1] R. Merlin, Solid State Commun. 102, 207 (1997).

[2] S. L. Johnson, M. Savoini, P. Beaud, G. Ingold, U. Staub, F. Carbone, L. Castiglioni, M. Hengsberger, and J. Osterwalder, Struct. Dyn. 4, 061506 (2017).

[3] T. K. Cheng, J. Vidal, H. J. Zeiger, G. Dresselhaus, M. S. Dresselhaus, and E. P. Ippen, Appl. Phys. Lett. 59, 1923 (1991).

[4] H. J. Zeiger, J. Vidal, T. K. Cheng, E. P. Ippen, G. Dresselhaus, and M. S. Dresselhaus, Phys. Rev. B 45, 768 (1992).

[5] G. A. Garrett, T. F. Albrecht, J. F. Whitaker, and R. Merlin, Phys. Rev. Lett. 77, 3661 (1996).

[6] M. Hase, K. Mizoguchi, H. Harima, S. Nakashima, M. Tani, K. Sakai, and M. Hangyo, Appl. Phys. Lett. 69, 2474 (1996).

[7] C. A. D. Roeser, M. Kandyla, A. Mendioroz, and E. Mazur, Phys. Rev. B 70, 212302 (2004).

[8] M. F. DeCamp, D. A. Reis, P. H. Bucksbaum, and R. Merlin, Phys. Rev. B 64, 092301 (2001).

[9] P. Beaud, S. L. Johnson, A. Streun, R. Abela, D. Abramsohn, D. Grolimund, F. Krasniqi, T. Schmidt, V. Schlott, and G. Ingold, Phys. Rev. Lett. 99, 174801 (2007).

[10] L. Rettig, J.-H. Chu, I. R. Fisher, U. Bovensiepen, and M. Wolf, Faraday Discuss. 171, 299 (2014).

[11] P. Beaud, S. L. Johnson, E. Vorobeva, U. Staub, R. A. De Souza, C. J. Milne, Q. X. Jia, and G. Ingold, Phys. Rev. Lett. 103, 155702 (2009).

[12] M. Eichberger, H. Schäfer, M. Krumova, M. Beyer, J. Demsar, H. Berger, G. Moriena, G. Sciaini, and R. J. Miller, Nature (London) 468, 799 (2010).

[13] W. Lu, M. Nicoul, U. Shymanovich, A. Tarasevitch, M. Kammler, M. Horn von Hoegen, D. von der Linde, and K. Sokolowski-Tinten, Mater. Res. Soc. Symp. Proc. 1230, MM03 (2010).

[14] T. Huber, S. O. Mariager, A. Ferrer, H. Schäfer, J. A. Johnson, S. Grübel, A. Lübcke, L. Huber, T. Kubacka, C. Dornes, C. Laulhe, S. Ravy, G. Ingold, P. Beaud,
J. Demsar, and S. L. Johnson, Phys. Rev. Lett. 113, 026401 (2014).

[15] P. Beaud, A. Caviezel, S. O. Mariager, L. Rettig, G. Ingold, C. Dornes, S.-W. Huang, J. A. Johnson, M. Radovic, T. Huber, T. Kubacka, A. Ferrer, H. T. Lemke, M. Chollet, D. Zhu, J. M. Glownia, M. Sikorski, A. Robert, H. Wadati, M. Nakamura et al., Nat. Mater. 13, 923 (2014).

[16] M. Trigo, P. Giraldo-Gallo, M. E. Kozina, T. Henighan, M. P. Jiang, H. Liu, J. N. Clark, M. Chollet, J. M. Glownia, D. Zhu, T. Katayama, D. Leuenberger, P. S. Kirchmann, I. R. Fisher, Z. X. Shen, and D. A. Reis, Phys. Rev. B 99, 104111 (2019).

[17] R. Yusupov, T. Mertelj, V. V. Kabanov, S. Brazovskii, P. Kusar, J. H. Chu, I. R. Fisher, and D. Mihailovic, Nat. Phys. 6, 681 (2010).

[18] S. Wall, D. Wegkamp, L. Foglia, K. Appavoo, J. Nag, R. F. Haglund, J. Stähler, and M. Wolf, Nat. Commun. 3, 721 (2012).

[19] R. E. Peierls, Quantum Theory of Solids (Clarendon, Oxford, UK, 1955).

[20] W. Fogle and J. H. Perlstein, Phys. Rev. B 6, 1402 (1972).

[21] G. Travaglini, P. Wachter, J. Marcus, and C. Schlenker, Solid State Commun. 37, 599 (1981).

[22] J. P. Pouget, B. Hennion, C. Escribe-Filippini, and M. Sato, Phys. Rev. B 43, 8421 (1991).

[23] A. Tomeljak, H. Schäfer, D. Städter, M. Beyer, K. Biljakovic, and J. Demsar, Phys. Rev. Lett. 102, 066404 (2009).

[24] R. Mankowsky, B. Liu, S. Rajasekaran, H. Y. Liu, D. Mou, X. J. Zhou, R. Merlin, M. Först, and A. Cavalleri, Phys. Rev. Lett. 118, 116402 (2017).

[25] W. J. Schutte and J. L. de Boer, Acta Crystallogr., Sect. B 49, 579 (1993).

[26] See Supplemental Material at http://link.aps.org/supplemental/ 10.1103/PhysRevB.99.220302 for details on the experimental setup and the data fitting process.

[27] L. D. Landau and E. M. Lifshitz, Statistical Physics, 2nd ed. (Pergamon, Oxford, UK, 1968). 